\title{
Considering Point-of-Care Electronic Medical Resources in Lieu of Traditional Textbooks for Medical Education
}

\author{
Courtney R. Adams, Michelle L. Kaufman, Courtney L. Snyder \\ Faculty: LaDonna S. Hale, PharmD and Michelle Wallace, MPAS, PA-C \\ Department of Physician Assistant, College of Health Professions
}

Within just 26 months, physician assistant students are transformed from relatively medically naive learners into licensed healthcare providers with a high level of autonomy. Didactic education traditionally relies on medical textbooks to fill knowledge gaps and clarify/deepen comprehension. However, clinicians rarely use textbooks to practice medicine. Instead, they use point-of-care electronic resources, drug information, and medical applications (eg, UpToDate, Clinical Pharmacology). Thus, PA students are also expected to demonstrate strong information literacy skills and proficiency with these resources during clinical rotations and as clinicians. Information literacy skills (the ability to recognize when information is needed and to identify, locate and effectively use information) require time and mentoring. Thus, PA students must rapidly gain knowledge and information literacy skills simultaneously. This article explores the use of POC resources in lieu of textbooks during didactic training to bridge the gap between classroom and clinical setting. (Published: Journal of Physician Assistant Education, 2015.) 